\title{
Desenvolvimento de tecnologia educativa para mães de crianças internadas em paliação não oncológica
}

\author{
Construction and validation of educational technology for mothers of hospitalized and \\ palliated children
}

Desarrollo de tecnología educativa para madres de niños con paliación no oncológica

Evilania de Souza Soares ${ }^{1 *}$, Vera Lucia Mendes de Paula Pessoa ${ }^{1}$, Raquel Sampaio Florêncio ${ }^{1}$, Mardenia Gomes Vasconcelos Pitombeira ${ }^{1}$, Virna Ribeiro Feitosa Cestari¹.

\section{RESUMO}

Objetivo: Construir e validar uma tecnologia educativa do tipo scrapbook direcionada para as mães de crianças internadas e paliadas. Métodos: Trata-se de um estudo metodológico desenvolvido em três etapas: revisão integrativa sobre os cuidados paliativos na pediatria; entrevistas semiestruturadas com as mães de crianças hospitalizadas iniciando a paliação e construção e validação do scrapbook em versão piloto. A estrutura foi delineada a partir da Pedagogia da Autonomia de Paulo Freire (1996). Resultados: A tecnologia teve seu conteúdo obtido por meio da revisão integrativa e das entrevistas maternas. Ambas sinalizaram para a necessidade das mães de se sentirem acolhidas no ambiente hospitalar onde acompanham seus filhos. De forma geral, o scrapbook apareceu como uma tecnologia interativa que necessita da intervenção materna. A avaliação das juízas, compilada em conteúdo e aparência, classificou a tecnologia educativa validada com um IVC de 0,80. Conclusão: Assim, o material construído foi considerado validado pelas experts na temática e pode vir a ser útil junto às equipes multidisciplinares de cuidados paliativos na pediatria.

Palavras-chave: Cuidados paliativos, Pediatria, Mães, Tecnologia educativa.

\begin{abstract}
Objective: To build and validate a scrapbook type educational technology directed to the mothers of interned and palliated children. Methods: This is a methodological developed in three stages: integrative review on palliative care in pediatrics; semi-structured interviews with the mothers of hospitalized children starting the palliation and construction and validation of the scrapbook in pilot version. The structure was outlined from the Pedagogy of Autonomy of Paulo Freire (1996). Results: The technology had its content obtained through the integrative review and the maternal interviews. Both signaled the need of mothers to feel welcomed in the hospital environment where they accompany their children. In general, the scrapbook appeared as an interactive technology that needs maternal intervention. The judges' evaluation, compiled in content and appearance, classified the educational technology validated with an CVI of 0.80 . Conclusion: Thus, the material constructed was considered validated by the experts in the subject and can come to be useful with the multidisciplinary teams of palliative care in pediatrics.
\end{abstract}

Keywords: Palliative care, Pediatrics, Mothers, Health technology.

\section{RESUMEN}

Objetivo: Construir y validar una tecnología educativa tipo álbum de recortes dirigida a las madres de niños detenidos y paliados. Métodos: Se trata de un estudio metodológico desarrollado en tres etapas: revisión integradora sobre cuidados paliativos en pediatría; entrevistas semiestructuradas con las madres de niños hospitalizados que inician la paliación y construcción y validación del cuaderno de recortes en una versión piloto. La estructura fue esbozada a partir de la Pedagogía de la Autonomía de Paulo Freire (1996). Resultados: El contenido de la tecnología se obtuvo a través de la revisión integradora y las entrevistas a las madres. Ambos indicaron la necesidad de que las madres se sientan acogidas en el entorno hospitalario donde acompañan a sus hijos. En general, el álbum de recortes apareció como una tecnología interactiva que

1 Universidade Estadual do Ceará (UECE), Fortaleza - CE. `E-mail: evilaniass@yahoo.com.br 
necesita la intervención materna. La evaluación de los jueces, recopilada en contenido y apariencia, clasificó la tecnología educativa validada con un IVC de 0,80. Conclusión: Así, el material construido se consideró validado por los expertos en el tema y puede llegar a ser útil con los equipos multidisciplinarios de cuidados paliativos en pediatría.

Palabras clave: Cuidados paliativos, Pediatría, Madres, Tecnología educativa.

\section{INTRODUÇÃO}

Os cuidados paliativos, conforme a Organização Mundial da Saúde (OMS), são uma abordagem que direciona a atenção igualmente aos familiares e aos pacientes com doenças potencialmente fatais e consideram os problemas paralelos como os psicossociais ou os espirituais. Esse conceito reflete 0 amadurecimento das discussões que ocorrem desde 1990, quando, pela primeira vez, a OMS o definiu (WHO, 2020).

Os países de baixa e média renda albergam cerca de $78 \%$ das 40 milhões de pessoas que necessitam de cuidados paliativos por ano, sendo que, desse quantitativo, aproximadamente $14 \%$ os recebem. No caso das crianças, $98 \%$ das que precisam de cuidados paliativos estão em países de baixa e média renda, com quase metade delas vivendo na África (WHO, 2020). A legislação brasileira, na Resolução n.. 41/2018, ressalta desde o conceito e os princípios até o financiamento dos cuidados paliativos no Sistema Único de Saúde (SUS) (BRASIL, 2018).

Em conformidade com a recomendação da oferta de cuidados paliativos na rede hospitalar, presente no artigo $5^{\circ}$ e inciso $\mathrm{V}$ da referida legislação, o hospital pediátrico de nível terciário e referência pública no estado do Ceará dispõe de duas equipes de cuidados paliativos, uma com atuação há cerca oito anos na Oncologia e a outra há três anos na Pediatria da Clínica Geral. E esta trouxe o carro chefe que interessou ao estudo: as mães de crianças e adolescentes hospitalizados por doenças não oncológicas e sem perspectiva de tratamento curativo.

A família (em alguns casos, os pais, e, não raro, somente a mãe) atua enquanto cuidadora principal da criança internada. Portanto, deve receber as devidas orientações tanto da equipe de assistência quanto da paliativista, visando à minimização de possíveis conflitos da ordem de entendimento e maximizar a cooperação mútua (VIDAL V, et al., 2019).

De acordo com Barros $F$ (2019), apesar de o paciente não se mostrar responsivo às terapêuticas de cura, as equipes precisam cultivar uma relação dialógica com os familiares. Nesse sentido, a conduta do profissional de saúde pode, por meio da utilização de recursos, diminuir a ansiedade nesses familiares, principalmente na genitora.

Sendo assim, o objetivo desta pesquisa foi construir e validar uma tecnologia educativa do tipo scrapbook para mães de crianças internadas e paliadas.

Entende-se, por tecnologia educativa, todos os conhecimentos necessários a serem utilizados em atividades de caráter educativo, levando em consideração a sua construção a partir do complexo contexto de vida do ser humano, suas experiências, bem como as expectativas diante do processo saúde-doença (WALL ML, 2001). São inumeráveis os tipos de tecnologia que objetivam transmitir ou compartilhar informações, tais como: vídeos, palestras, dinâmicas de grupos, dramatizações, etc. Pode-se incluir neste rol os manuais, os folders, as cartilhas, dentre outros, que se adequam às necessidades de cada objetivo ou meta.

Essa prática educativa não está vinculada a apenas uma profissão específica, mas tende a se adequar aos mais diversos grupos, uma vez que valoriza os indivíduos envolvidos em todo o processo, desde a construção até a sua finalização, como é o caso de tecnologias como o scrapbook, que não somente estimulam a participação efetiva da população-alvo, mas tornam essa participação como parte do processo de sua confecção. 


\section{MÉTODOS}

Trata-se de estudo do tipo metodológico, pois almeja a construção e a validação de instrumento metodológico (POLIT DF, et al., 2019). Conforme pode ser observado na Figura 1, Paulo Freire e a Pedagogia da Autonomia (1996) configuraram o delineamento da tecnologia sugerida, pois enfatizam os sujeitos e suas experiências (Figura 1).

Figura 1 - Fluxograma das fases do estudo.

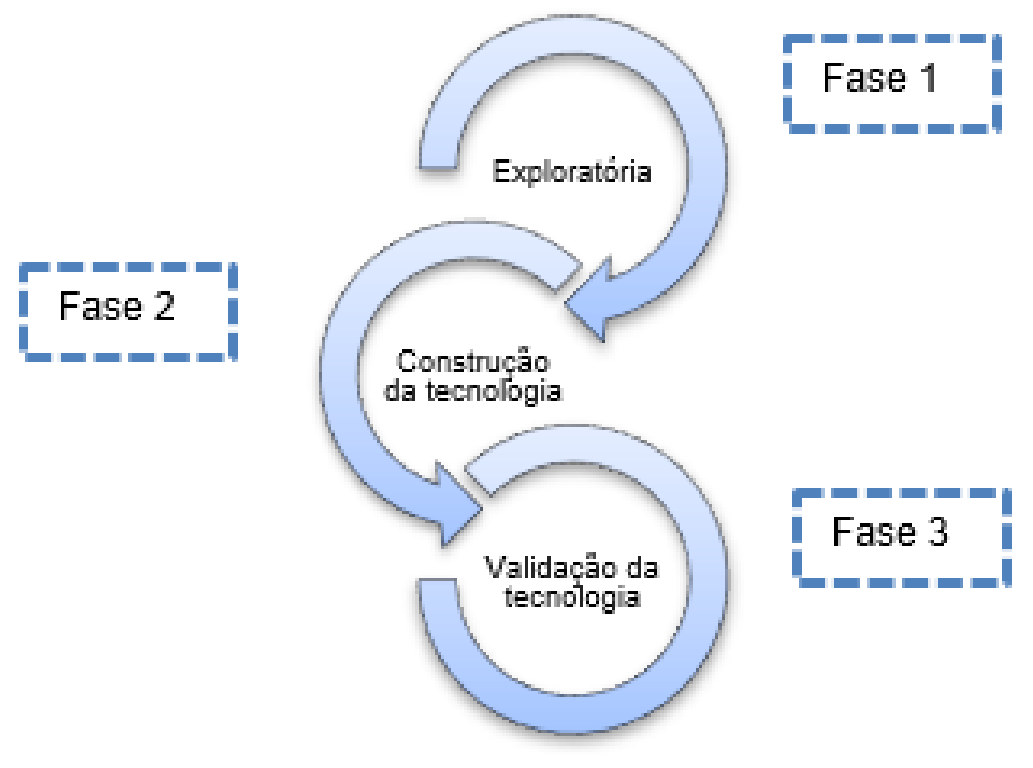

Fonte: Soares ES, et al., 2021. Com base em Echer IC, 2005.

Em busca de compor o conteúdo da tecnologia em saúde, foi realizada inicialmente uma revisão integrativa (RI), seguida por entrevistas semiestruturadas com mães de pacientes internados e em paliação. A revisão integrativa ocorreu de janeiro a março de 2020, nas bases de dados da área da saúde: Banco de Dados em Enfermagem (BDENF), Literatura Latino-Americana e do Caribe em Ciências de Saúde (LILACS), Índice Bibliográfico Español de Ciencias de la Salud (IBECS) (via Biblioteca Virtual em Saúde - BVS), no portal National Library of Medicine and National Institute of Health (PubMed), Science Direct e Cumulative Index to Nursing and Allied Health Literature (CINAHL) (via Portal CAPES) e na biblioteca eletrônica Scientific Electronic Library Online (SciELO).

Com a análise dos dez artigos selecionados conforme o protocolo Preferred Reporting Items for Systematic Reviews and Meta-Analyses (PRISMA), obteve-se como resultado que a maioria dos estudos versava sobre cuidados paliativos e câncer (MOHER D, et al., 2015). Percebeu-se também que, na pediatria, o entendimento dos familiares, principalmente das genitoras que acompanhavam o filho no hospital, sobre o cuidado paliativo ofertado ao filho precisa de atenção significativa. Além disso, outras necessidades maternas demandam dos cuidados dos profissionais de saúde.

Os critérios adotados para a inclusão das mães no estudo foram aceitar participar e estar acompanhando o filho internado e em início de paliação. A coleta dos dados ocorreu por meio de entrevistas semiestruturadas com as respectivas mães, as quais foram realizadas em hospital público, pediátrico, de nível terciário e referência no estado do Ceará. As 11 participantes que aceitaram o convite expressaram a sua lida no âmbito hospitalar acompanhando o filho paliado e descreveram os caminhos trilhados por elas e pelos filhos, que se desenrolaram a partir das limitações da doença.

Sob o ponto de vista materno, essas mães revelaram o processo íntimo de desconstrução do filho idealizado para a convivência com o filho real e toda a trajetória nos ambientes hospitalares. Em suma, a 
relação com os profissionais de saúde, com as outras mães, com os novos termos da área da saúde que precisaram aprender e com o cuidar do filho por esses profissionais, representando a rotina diária dessas mães. Destaca-se que o conteúdo religioso esteve presente na maior parte das falas.

Após a análise das entrevistas, com o auxílio do software Iramuteq ${ }^{\circledR}$, vislumbrou-se que a necessidade materna sinalizou para o acolhimento. Assim, buscou-se na Pedagogia da Autonomia (1996), de Paulo Freire, o fundamento pedagógico para a tecnologia elaborada para as mães.

Então, pelas experiências cotidianas do binômio mãe e filho internado, expressas com a revisão integrativa e pelas sutilezas apresentadas nos depoimentos maternos, optou-se por uma tecnologia do tipo scrapbook. Ou melhor, pensou-se em construir um instrumento com formato interativo para que as mães pudessem se expressar e registrar sua compreensão do vivido, pensado ou esperançado.

O scrapbook não seria um álbum ou um diário convencional, mas talvez a união das duas concepções. No diário, a ocorrência maior está na escrita; e, no álbum, nos registros fotográficos. O scrapbook, ao unir as duas ideias, amplia a possibilidade de resgatar o contexto vivenciado e registrá-lo tanto de forma ilustrada quanto descritiva, podendo ainda ser anexados, por exemplo: um desenho do pôr do sol a lápis de cor, uma embalagem de um produto utilizado ou parte dela, uma frase dita, um poema inteiro ou um verso, uma letra de música e o que a criatividade ou o que o momento no dia a dia decidirem por manter (HIRATOMI L, et al., 2011; DO CARMO AFC, et al., 2013).

Dessa forma, o scrapbook foi estruturado a partir da questão da autonomia de Paulo Freire (1996) com a escolha da linguagem e também com a aparência convidativa à intervenção com espaços em branco entre mensagens e figuras. Pensou-se inicialmente, para a versão inicial, em 16 páginas de papel A4 com uma dobra, contendo frases inspiradas a partir da revisão integrativa e das entrevistas maternas, bem como ilustrações de livre acesso no Google e lacunas em branco entre ambas.

\section{Validação do Scrapbook}

A validação de conteúdo e de aparência foi efetuada de forma compilada por uma amostra do tipo conveniência, composta por dez avaliadores que atenderam aos critérios de inclusão adaptados por Joventino ES (2010), que foram: possuir título de doutor, 4 pontos; tese na área de cuidados paliativos, 2 pontos; mestre, 3 pontos; dissertação na área do constructo, 2 pontos; artigos publicados na área referida, 2 pontos; prática profissional na área, 2 pontos; e especialização na área, 2 pontos. Assim, os referidos juízes especialistas alcançaram uma pontuação mínima de 5 pontos.

Os avaliadores precisaram responder ao instrumento do tipo questionário marcando um " $x$ " na resposta que exprimia o seu exame alusivo àquele subitem. Para tanto, foi utilizada a Escala Tipo Likert adaptada da proposta de Oliveira MS (2006), com numeração de 1 a 4, com a equivalência dos termos: 1 = "discordo", 2 = "discordo parcialmente", 3 = "concordo parcialmente" e 4 = "concordo".

O instrumento continha cinco dimensões para serem avaliadas em conteúdo e aparência: Objetivos, Organização, Estilo da escrita, Aparência e Motivação. Estavam subdivididas em 22 subitens. Para cada item havia um espaço disponível para comentários e sugestões, bem como ao final do questionário.

A determinação do nível de concordância entre os especialistas foi calculada utilizando-se o Índice de Validade de Conteúdo (IVC) para cada um dos subitens e para o conjunto total de itens do instrumento. $O$ scrapbook foi avaliado em conteúdo e aparência, de modo que, conforme Polit DF, et al. (2019), quanto mais próximo do valor 1,00 mais forte será o nível de confiabilidade. Um valor de 0,78 ou superior confere a evidência em grau de satisfatoriedade para a validade de conteúdo. Alexandre NMC e Coluci MZO (2011) também recomendam que o valor do nível de concordância entre os juízes não pode ficar abaixo de 0,78.

\section{Aspectos éticos}

A pesquisadora apresentou o Termo de Consentimento Livre e Esclarecido (TCLE) e os objetivos da pesquisa tanto para o público-alvo quanto para os juízes especialistas, ambos voluntários da pesquisa, além de terem sido descritos os riscos e os benefícios para todos os participantes. O estudo seguiu todos os 
critérios da Resolução n. ${ }^{-466 / 12}$ do Conselho Nacional de Saúde (CNS) a respeito da ética em trabalhos de pesquisas com seres humanos, sendo aprovado com o Parecer $n .-\stackrel{3}{3.695 .881}$. Toda a coleta de dados ocorreu durante $o$ ano de 2020.

\section{RESULTADOS}

\section{A construção do scrapbook}

As mensagens que constam no scrapbook foram pensadas para serem de fácil compreensão pelas mães e ele possui espaços livres à disposição para que elas preencham com quaisquer pensamentos, dúvidas ou uma etiqueta de medicamento e até mesmo fotos. A Pedagogia da Autonomia, de Freire P (1996), afirma que o adulto difere da criança por perceber o que lhe faz falta e necessita apreender conforme os seus conhecimentos prévios.

Os scrapbooks trazem uma sistemática de complementaridade entre os elementos como textos e imagens diversas, desde as desenhadas até as anexadas, ou seja, a criatividade e a liberdade de expressão prevalecem no momento de preenchê-los. Nessa perspectiva, o scrapbook construído em versão preliminar foi planejado a partir da revisão integrativa e das categorias decorrentes das entrevistas: o convívio com o filho real versus o filho imaginado; o ambiente hospitalar e os cuidados paliativos.

Na versão inicial, o scrapbook foi intitulado Mães e filhos paliados em contexto hospitalar. Desde a capa, encontram-se opções indicativas para que a mãe as preencha. Os espaços em branco equivalem a um convite à prática, abrangendo desde os dados principais do filho, como nome e data de nascimento, até os complementos das figuras que faltam cor. Também foi levado em consideração o público do SUS, por ser diverso e flutuar entre os níveis de escolaridade. Para tanto, pensou-se que a equipe de cuidados paliativos, ao identificar aquelas mães que apresentem maior necessidade de apoio para lidar com o scrapbook, possa oferecer explanação cuidadosa e outros materiais, bem como ofertar, caso haja interesse, o auxílio para a sua composição.

\section{Processo de validação}

Todos os juízes foram do sexo feminino. A média de idade foi de 43,9 anos, variando de 28 a 63 anos, com desvio-padrão (DP) de $\pm 12,11$ anos. Dentre as profissões, ponderou-se que ouve uma diversidade de profissionais, sendo que a medicina teve quatro (40\%); a psicologia, três (30\%); a enfermagem, dois (20\%) e um $(10 \%)$ profissional do serviço social.

Conforme descrito na Tabela 1, a média quanto ao tempo de formação foi de 18,9 anos (DP \pm 13 anos). Com relação à titulação acadêmica, uma possuía o título de pós-doutorado (10\%), uma doutora (10\%), seis mestras $(60 \%)$ e duas especialistas (20\%). Percebeu-se que sete juízas $(70 \%)$ exerciam seu trabalho na área de cuidados paliativos na pediatria e nove (90\%) tinham publicação na referida área de interesse e, também, em outras correlatas (Tabela 1). 
Tabela 1 - Índice de Validade de Conteúdo e Teste Binomial dos itens de avaliação do scrapbook.

\begin{tabular}{|c|c|c|c|}
\hline \multicolumn{2}{|c|}{ Itens/subitens } & IVCia & $\mathbf{p}^{\mathbf{b}}$ \\
\hline \multicolumn{2}{|c|}{ Objetivos } & 0,68 & \\
\hline 1) & O texto da apresentação do scrapbook está claro e objetivo? & 0,5 & 0,078 \\
\hline 2) & $\begin{array}{l}\text { O scrapbook, enquanto possibilidade de construção manual pelas mães, } \\
\text { atende aos seus objetivos? }\end{array}$ & 0,7 & 0,474 \\
\hline 3) & $\begin{array}{l}\text { Irá ajudar no cotidiano das vivências maternas no ambiente hospitalar } \\
\text { enquanto acompanha o(a) filho(a) internado(a)? }\end{array}$ & 0,8 & 0,001 \\
\hline 4) & $\begin{array}{l}\text { Você acha que o instrumento ajudará a equipe de cuidados paliativos a } \\
\text { dialogar sobre os cuidados paliativos com as mães? }\end{array}$ & 0,6 & 0,020 \\
\hline 5) & O scrapbook remete à autonomia proposta na pedagogia freiriana? & 0,8 & 0,526 \\
\hline \multicolumn{2}{|c|}{ Organização } & 0,77 & \\
\hline 1) & A capa indicativa dos dados do filho está adequada? & 0,8 & 0,526 \\
\hline 2) & Os tópicos têm uma sequência lógica? & 0,9 & 0,244 \\
\hline 3) & Há coerência entre as frases e as imagens? & 0,8 & 0,526 \\
\hline 4) & O conteúdo está apropriado? & 0,6 & 0,020 \\
\hline 5) & O número de páginas está adequado? & 1,0 & 0,056 \\
\hline 6) & Os temas das frases retratam aspectos importantes? & 0,5 & 0,078 \\
\hline \multicolumn{2}{|c|}{ Estilo da escrita } & 0,90 & \\
\hline 1) & A escrita está em estilo adequado? & 0,8 & 0,526 \\
\hline 2) & O texto é interessante? O tom é amigável? & 0,9 & 0,001 \\
\hline 3) & O vocabulário é acessível? & 0,9 & 0,244 \\
\hline 4) & O texto está claro e coerente? & 1,0 & 0,056 \\
\hline \multicolumn{2}{|c|}{ Aparência } & 0,87 & \\
\hline 1) & As páginas parecem organizadas? & 1,0 & 0,056 \\
\hline 2) & As ilustrações são simples e suficientes? & 0,9 & 0,244 \\
\hline 3) & As ilustrações servem para complementar os textos? & 0,7 & 0,474 \\
\hline 4) & As ilustrações servem para estimular a expressividade materna? & 0,9 & 0,526 \\
\hline \multicolumn{2}{|c|}{ Motivação } & 0,77 & \\
\hline 1) & O material é apropriado para você? & 0,5 & 0,078 \\
\hline 2) & O conteúdo do scrapbook apresentou-se de forma lógica? & 0,9 & 0,244 \\
\hline 3) & $\begin{array}{l}\text { A mãe sente-se convidada à interação? Os espaços em branco são sugestivos } \\
\text { ou indicativos de complementação? }\end{array}$ & 0,9 & 0,244 \\
\hline \multicolumn{2}{|c|}{ IVC total } & 0,80 & - \\
\hline
\end{tabular}

Legenda: $\mathrm{a}=$ Índice de Validade de Conteúdo do item; $\mathrm{b}=$ Teste Binomial.

Fonte: Soares ES, et al., 2021. 
Verificou-se que, dos 22 subitens, 19 foram avaliados com Índices de Validade de Conteúdo (IVC) bons e excelentes, porém três tiveram IVC de 0,5 , que é abaixo do que preconiza a literatura $(\leq 5,9)$. No Teste Binomial, houve discrepância entre as juízas acerca da pontuação de quatro subitens: objetivos, organização, estilo da escrita e motivação.

No questionário avaliador, também foi solicitado às juízas que comentassem sobre o scrapbook, no intuito de melhorá-lo (Quadro 1). Elas consideraram que a tecnologia educativa proposta, conforme se observa no Quadro 2, é interessante e válida para ser implementada ou estar disponível no serviço de internação hospitalar, pois, inclusive, pode facilitar as manifestações maternas.

Quadro 1 - Comentários e sugestões das juízas sobre o scrapbook.

\begin{tabular}{|c|c|}
\hline Juiz & Comentários/sugestões \\
\hline J1 & $\begin{array}{l}\text { No scrapbook não há espaço para que mães não cristãs possam se expressar; é excludente; } \\
\text { Incluir dados relacionados ao filho e mais espaço para registros da criança pela mãe, não apenas } \\
\text { de fotos, mas de outras recordações; } \\
\text { Inserir mais espaços para os registros maternos de seu tempo internada; } \\
\text { Tornar o texto mais inclusivo para que as mães se sintam efetivamente amparadas. }\end{array}$ \\
\hline J2 & $\begin{array}{l}\text { Inserir introdução para explicar o que é o material e para que serve; } \\
\text { Não limitar por religião; } \\
\text { Reescrever a frase ideia expressa na página 07, sobre a presença materna, pois caso precise se } \\
\text { ausentar, pode gerar sentimento de culpa; } \\
\text { Inserir cores mais vivas; } \\
\text { Realizar validação com as próprias mães por meio de grupo focal ou dinâmicas em grupos. }\end{array}$ \\
\hline J3 & $\begin{array}{l}\text { Inserir espaço para data de admissão hospitalar; } \\
\text { Ampliar o material para várias religiões; } \\
\text { Retirar a imagem da mamadeira; } \\
\text { Substituir o termo holístico por outro mais simples; } \\
\text { Deixar claro que o material se restringe às mães de recém-nascidos/lactentes. }\end{array}$ \\
\hline J4 & mas; \\
\hline J5 & s no diminutivo; \\
\hline J7 & $\begin{array}{l}\text { dimensão do que são os cuidados paliativos e o tipo } \\
\text { er; } \\
\text { eligiosas. }\end{array}$ \\
\hline J8 & $\begin{array}{l}\text { Manter o documento todo em português; } \\
\text { Substituir a palavra scrapbook; } \\
\text { Retirar as frases motivacionais. }\end{array}$ \\
\hline J9 & $\begin{array}{l}\text { Aclarar o objetivo; } \\
\text { Inserir um espaço para o nome do pai na capa; } \\
\text { O conteúdo religioso pode gerar desconforto para famílias não religiosas; } \\
\text { Deixar mais espaços para dúvidas e preocupações; } \\
\text { Modificar o título de forma a deixar claro que o material é para os pais de filhos paliados em } \\
\text { contexto hospitalar; } \\
\text { Alterar a figura na página final, pois se a mãe não conseguir amamentar o filho, poderá gerar } \\
\text { sentimentos de incapacidade; } \\
\text { Na página 12, deixar mais espaço para que os pais possam escrever o nome das pessoas } \\
\text { da equipe dos cuidados paliativos. }\end{array}$ \\
\hline J10 & $\begin{array}{l}\text { Modificar as cores e letras para diferenciar falas das mães e dos profissionais; } \\
\text { Inserir dados familiares (pais, irmãos, avós); } \\
\text { Reescrever termos de difícil compreensão, como cuidados holísticos; } \\
\text { Abordar a espiritualidade de forma mais ampla. }\end{array}$ \\
\hline
\end{tabular}

Legenda: Os itens grifados em negrito foram levados em consideração para as modificações mais significativas.

Fonte: Soares ES, et al., 2021. 
A maior parte dos detalhes observados pelas juízas foi analisada e considerada; entretanto, outros não, pois nas ocorrências em que as entrevistas, de antemão realizadas, apontaram para a importância daquele tópico ou daquele conteúdo da tecnologia, a pesquisadora preferiu mantê-los. Também por compreender que o scrapbook considera a intenção materna: o que essa mãe quer? Quer sentir-se de alguma forma acolhida, motivada, além de ter o direito de expressar temores e esperanças sobre a vida do filho. Então necessita, para tanto, de assistência e encorajamento.

Quadro 2 - Aspectos positivos do scrapbook.

\begin{tabular}{|c|l|}
\hline Juiz & \multicolumn{1}{|c|}{ Aspectos positivos } \\
\hline J1 & $\begin{array}{l}\text { Proposta válida como uma forma da mãe expressar seus sentimentos, ser } \\
\text { elaborar a resiliência; } \\
\text { A linguagem está adequada para mães de diferentes classes sociais; } \\
\text { Os espaços são convidativos. }\end{array}$ \\
\hline $\mathrm{J} 2$ & Tópicos e quantidade de páginas excelentes. \\
\hline $\mathrm{J} 4$ & $\begin{array}{l}\text { O scrapbook será de grande utilidade para mães com filhos em situação } \\
\text { de paliação. }\end{array}$ \\
\hline $\mathrm{J} 5$ & $\begin{array}{l}\text { Proposta excelente; } \\
\text { Material com potencial para suporte afetivo e de construção de vínculo das } \\
\text { mães/pais com a equipe e com o projeto terapêutico paliativo. }\end{array}$ \\
\hline J9 & $\begin{array}{l}\text { Material interessante para facilitar a expressão das vivências dos pais que } \\
\text { têm filhos internados em cuidados paliativos. }\end{array}$ \\
\hline
\end{tabular}

Fonte: Soares ES, et al., 2021.

Pela interpretação da maioria dos especialistas, o teor espiritual e/ou referente à religiosidade deveria ser repensado e até excluído da tecnologia. No entanto, apesar do consenso encontrado, assumiu-se que o aludido conteúdo é relevante a partir da revisão integrativa e dos diálogos maternos. Enfim, houve concordância em acatar parte do recomendado pelas juízas uma vez que se padronizou o scrapbook, reduziram-se algumas ilustrações, trocaram-se outras e também os textos foram modificados.

\section{DISCUSSÃO}

Os dados obtidos na fase 1 ou exploratória trouxeram algumas reflexões acerca da temática abordada por pesquisas, bem como as entrevistas que foram realizadas com as mães. Essa dupla coleta permitiu vislumbrar, de um lado, o que traziam os trabalhos científicos; e, do outro, conhecer as demandas maternas.

Nessa primeira fase, chegou-se à constatação de que a indicação de paliação ao filho internado, conforme os autores Pöder U, et al. (2008) e Sanches MVP, et al. (2014), ratifica a ideia de que os pais, em sua grande maioria, sinalizam por uma atenção especial ou diferenciada por não se sentirem preparados para outra perspectiva ou etapa do tratamento que não a curativa. As autoras compreenderam a dimensão nova que se descortinava para ambos: equipe e familiares, que era a necessidade de algum tipo de preparação para conduzir esse processo.

A partir da análise de diversos estudos, nota-se que os autores perceberam que a formação, capacitação ou especialização em cuidados paliativos seria basilar para os profissionais de saúde oferecerem o suporte adequado aos familiares (COSTA MADJ, et al., 2016; MCNAMARA LC, et al., 2020; DOUMIT MAA, et al., 2019; SANCHES MVP, et al., 2014; BOSQUET-DEL MORAL LBD, et al., 2012; COSTA MADJ, et al., 2018; ATOUT M, et al., 2019). E que, dentre estes, é a figura materna que requer maior atenção, como complementa os estudos de Bosquet-del Moral LBD, et al. (2012). Portanto, é a mãe que, em boa parte dos casos, fica acompanhando o filho internado e demanda atenção biopsicossocial e espiritual por parte da equipe multiprofissional.

Ainda nessa fase exploratória, as entrevistas maternas trouxeram à tona os elementos de seus universos, tais como: o filho, em primeiro lugar; o seu papel de mãe; o médico e a equipe; o hospital e sua rotina; a casa e o marido; as outras acompanhantes, que se tornaram amigas; a religiosidade e os cuidados paliativos. 
Na segunda fase, o scrapbook foi elaborado a partir de frases breves, ilustrações e espaços em branco sinalizando a possibilidade para a expressão e interação materna. A ideia dessa tecnologia foi sugestão das integrantes da banca de qualificação desta pesquisa, que se coadunou com os discursos das participantes. Divisou-se a ideia de que, longe de familiares e do ambiente doméstico, talvez as mães buscassem por uma aproximação, então se pensou em um espaço de participação e partilha por parte delas.

Na terceira fase, a versão preliminar foi submetida à análise de profissionais com expertise na área e que avaliaram de forma compilada o conteúdo e a aparência do scrapbook. Essa tecnologia pode ser inserida no rol de recursos da equipe multidisciplinar de cuidados paliativos (como consta no Quadro 2) por ser capaz de oferecer às mães uma forma possível para se expressarem. Faz-se necessário esclarecer que não se teve a pretensão de que o material proposto pudesse ser generalizado aos demais familiares do paciente pediátrico, mesmo sabendo-se da necessidade de cuidados integrais também a estes, como preconizam os cuidados paliativos

A versão final do scrapbook permaneceu com o mesmo título e quantidade de páginas. O tamanho foi modificado para atender às sugestões das juízas, tendo ficado ligeiramente maior que A4 e menor que A3, com uma dobra. As figuras estão propositadamente sem cor. Algumas frases instigam complemento; por exemplo, em uma página consta o seguinte: “minha dúvida é... minha preocupação é...”. Em outra, já aparece uma sugestão: "vou colar uma foto inesquecível aqui...". A seguinte, traz a palavra "hoje" acompanhada por linhas em branco e ladeada por uma figura de uma mulher segurando o seu bebê. Assim, concluiu-se o scrapbook.

\section{CONCLUSÃO}

O resultado alcançado nesta pesquisa, por meio da validação de aparência e de conteúdo do scrapbook, pelo grupo de juízas experts na temática, mostrou um nível satisfatório de validação. Reconhece-se, entretanto, a limitação do estudo pela não validação com o público-alvo. Constatou-se que o material produzido pode vir a ser utilizado pelas equipes hospitalares com fins de auxiliar o processo de assistência materna quando da internação do filho paliado. Além disso, pode servir também de incentivo para outros estudos e novas propostas de tecnologias ou reflexões sobre cuidados paliativos, especialmente na pediatria. Por fim, o scrapbook pode ser acessado de modo on-line no site do Mestrado Profissional em Saúde da Criança e do Adolescente da Universidade Estadual do Ceará.

\section{REFERÊNCIAS}

1. ALEXANDRE NMC, COLUCI MZO. Validade de instrumento e medidas. Ciência \& Saúde Coletiva, 2011; 16(7): 306168.

2. ATOUT M, et al. The practice of mutual protection in the care of children with palliative care needs: a multiple qualitative case study approach from Jordan. Journal of Pediatric Nursing, 2019; 45: e9-e18.

3. BARROS F. Cirurgia paliativa em pediatria. In: BARBOSA SMM, ZOBOLI I, IGLESIAS SBO. (Orgs.). Cuidados paliativos na prática pediátrica. Rio de Janeiro: Atheneu, 2019; p. 189-197.

4. BRASIL. Ministério da Saúde. Resolução n.․ 41, de 31 de outubro de 2018. Dispõe sobre as diretrizes para a organização dos cuidados paliativos, à luz dos cuidados continuados integrados, no âmbito do Sistema Único de Saúde (SUS). Diário Oficial da União, Brasília, DF, n 225, 23 nov. 2018. Seção 1, p. 276.

5. COSTA MADJ, et al. Desvelando a experiência de mães de crianças com câncer. Revista de Enfermagem do Centro Oeste Mineiro, 2016; 6(1): 2052-65.

6. COSTA MADJ, et al. Experiências de mães de crianças com câncer em cuidados paliativos. Revista de Enfermagem UFPE, 2018; 12(5): 1355-64.

7. DO CARMO AFC, et al. Aspectos tecnológicos no desenvolvimento de um objeto de aprendizagem com acessibilidade: a experiência com o scrapbook. Colloquium Exactarum, 2013; 3(2): 93-104.

8. DOUMIT MAA, et al. Spirituality among parents of children with cancer in a Middle Eastern country. European Journal of Oncology Nursing, 2019; 39: 21-27.

9. ECHER IC. Elaboração de manuais de orientação para o cuidado em saúde. Revista Latino-Americana de Enfermagem, 2005; 13(5): 754-7.

10. FREIRE P. Pedagogia da autonomia: saberes necessários à prática educativa. 25. ed. São Paulo: Paz e Terra, 1996; $166 p$. 
11. HIRATOMI L, et al. Guia do professor scrapbook: a arte de decorar álbuns de fotografias. In: UNIVERSIDADE ESTADUAL PAULISTA. Caderno de formação: formação de professores didática dos conteúdos. São Paulo: Cultura Acadêmica, 2011; p. 46-52.

12. JOVENTINO ES. Construção e validação de escala para mensurar a autoeficácia materna na prevenção da diarreia infantil. Dissertação (Mestrado em Enfermagem) - Faculdade de Farmácia, Odontologia e Enfermagem. Universidade Federal do Ceará, Fortaleza, 2010; 249 p.

13. MCNAMARA LC, et al. "Dê-lhes a porta, mas não a empurre": atitudes da família em relação aos cuidados espirituais conduzidos por médicos em medicina paliativa pediátrica. Journal of Religion \& Health, 2020; 59: 2899-2917.

14. MOHER D, et al. Principais itens para relatar Revisões Sistemáticas e Meta-análises: A recomendação PRISMA. Tradução: Taís Freire Galvão e Thais de Souza Andrade Pansani; retro-traduzido por: David Harrad. Revista Epidemiologia e Serviços de Saúde, 2015; 24(2): 335-42.

15. BOSQUET-DEL MORAL LBD, et al. Vivencias y experiencias de duelo en madres tras un proceso de enfermedad oncológica de sus hijos. Medicina Paliativa, 2012; 19(2): 64-72.

16. OLIVEIRA, M. S. Autocuidado da mulher na reabilitação da mastectomia: estudo de validação de aparência e conteúdo de uma tecnologia educativa. Dissertação (Mestrado em Enfermagem) - Universidade Federal do Ceará, Fortaleza, 2006; 115 p.

17. PÖDER U, et al. Posttraumatic stress disorder among parents of children on cancer treatment: a longitudinal study. Psychooncology, 2008; 17(5): 430-37.

18. POLIT DF, et al. Fundamentos de pesquisa em enfermagem: avaliação de evidências para a prática da enfermagem. 9. ed. Porto Alegre: Artmed, 2019; 453 p.

19. SANCHES MVP, et al. Crianças e adolescentes com câncer em cuidados paliativos: experiência de familiares. Revista Brasileira de Enfermagem, 2014; 67(1): 28-35.

20. VIDAL V, et al. A importância de uma equipe de cuidados paliativos pediátricos. In: BARBOSA SMM, et al. (Orgs.). Cuidados paliativos na prática pediátrica. Rio de Janeiro: Atheneu, 2019; p. 77-81.

21. WALL ML. Tecnologias educativas: subsídios para a assistência de enfermagem em grupos. Goiânia: AB, 2001.

22. WORLD HEALTH ORGANIZATION. Fact sheets. Palliative care. Geneva: WHO, 2020. 